
3 Research Square
Preprints are preliminary reports that have not undergone peer review.
They should not be considered conclusive, used to inform clinical practice,
or referenced by the media as validated information.

\title{
The alteration of metabolic profiling in the second trimester of GDM and high risk pregnancy, and a nomogram for predicting macrosomia
}

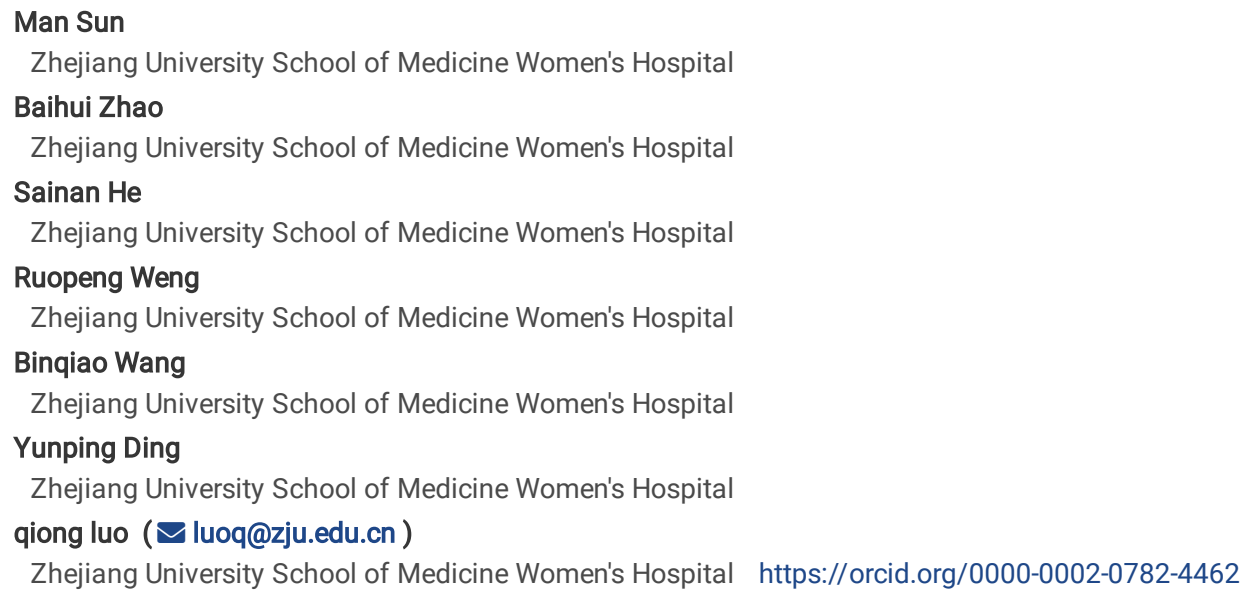

Zhejiang University School of Medicine Women's Hospital

Baihui Zhao

Zhejiang University School of Medicine Women's Hospital

Sainan He

Zhejiang University School of Medicine Women's Hospital

Ruopeng Weng

Zhejiang University School of Medicine Women's Hospital

Binqiao Wang

Zhejiang University School of Medicine Women's Hospital

Yunping Ding

Zhejiang University School of Medicine Women's Hospital

qiong luo ( $\sim$ luoq@zju.edu.cn )

Zhejiang University School of Medicine Women's Hospital https://orcid.org/0000-0002-0782-4462

\section{Research}

Keywords: Metabolomics, carnitine, GDM, macrosomia, pregnancy

Posted Date: December 28th, 2019

DOI: https://doi.org/10.21203/rs.2.19741/v1

License: (c) (i) This work is licensed under a Creative Commons Attribution 4.0 International License. Read Full License 


\section{Abstract}

Objective

Dyslipidemia in the second trimester and associated gestational diabetes are increasing worldwide. Carnitine plays a key role in lipid metabolism. We aim to describe metabolic profiling in the second trimester based on carnitine related metabolomics in GDM and high risk pregnancy, and to find the potential risk factors in GDM and candidate metabolites for diagnosing GDM induced macrosomia.

Methods

We have randomly investigated 450 pregnant women and their neonates in this retrospective study and 56 (12.4\%) GDM cases were diagnosed. We used LCMS/MS performing metabolic profiling about 12 amino acids and 31 acylcarnitines (containing $\mathrm{C} 0$ ) to assess circulating metabolites concentration in different subgroup according maternal and newborn clinical characteristic. We also calculated the correlation coefficient between maternal and newborn. GDM potential metabolic risk factors were screened by PLS-DA. Multivariate regression analyses were used in identifying independent risk factors for GDM and macrosomia. Based on these carnitine-related factors, a nomogram for estimating macrosomia was developed.

Results

We found 14 AA (Ala, Arg, Met, BCAA, AAA) and AC (C0, C2, C3, C4DC+C5OH, C5, C16, C18, C18:1) were increased in Age > 35 group, BMI $\geq 30$, weight gain > $20 \mathrm{~kg}$ group, using assistant reproductive technology group, but the level of $\mathrm{CO}$, Gly were decreased. In fetal clinical data, we obtained AA and AC level in fetuses are higher than their mothers and the metabolic trend was similar with maternal result. PLS-DA showed 15 metabolism(C0, LEU+ILE+PRO-OH, Phe, C18, TYR, etc)play main roles in class separation of GDM. Multivariate analysis showed pre-pregnancy BMI, weight gain, LEU+ILE+PRO-OH, TYR, $\mathrm{C} 0$ /acylcarnitine, C0, C3, C16, C18 are independent risk factors associated with GDM. Finally, we developed a nomogram predicting macrosomia based on carnitine-related metabolic variables.

Conclusion

Metabolomics was proved as a powerful tool in identifying the metabolic alteration during the second trimester. These metabolic risk factors in GDM may help understanding of the underlying biochemical pathology of GDM and help physician diagnosing macrosomia.

\section{Introduction}

Pregnancy is a complex process accompanied with substantial changes in lipid metabolism. In the second trimester, maternal metabolism is an anabolic state combined with increasing lipogenesis, accelerating maternal fat stores. [1] In the third trimester, there is a catabolic state breakdown in fat depot for fetal growth. Dyslipidemia during the second trimester is associated with metabolic disorder (GDM, hyperlipemia, hypertension) and adverse neonate outcomes (macrosomia).[2] Carnitine and related amino acid (AA) play key roles in fatty acid metabolism. [3] So, it is essential to investigate carnitine related metabolomics in the second trimester and find metabolomic risk factors for metabolic disorder diseases such as GDM.

GDM is a common metabolic disorder during pregnancy, occurs in approximately 8-10\% of pregnancies in global. [4] Pregnant women with GDM are more prone to hypertension and metabolic syndrome. The risks to fetus include macrosomia, respiratory distress syndrome, childhood obesity and T2DM in adults. [5] The main causes of GDM are still undefined, a number of studies have reported that the serum levels of branched-chain and aromatic amino acids (BCAAs and AAAs), including leucine, isoleucine, valine, phenylalanine, and tyrosine, are significantly different among lean, obesity, and diabetes, and closely correlated to insulin resistance, which highlighted their potential impact on diabetes diagnosis and risk assessment. [6] Carnitine worked as a critical role in energy metabolism of transporting long-chain fatty acid from the cytosol into the mitochondria. [7] Some studies propose free carnitine and acetyl carnitine (AC) are decreased in pregnancy at first trimester compared with non-pregnancy.[8] However, studies of metabolic profiling about carnitine in second trimester among diverse pregnant women are rare. Herein, we provide a study of metabolism of carnitine and related AA about normal, high risk pregnancy and GDM, and investigate the potential risk factors in GDM.

Fetal macrosomia is clearly related with GDM. Macrosomia can induce multiple perinatal complication, such as shoulder dystocia, brachial plexus injury. [9] Some surveys have found beta-alanine, phenylalanine, 4-aminobutyric acid shows different levels of cord blood with macrosomia and neonates with normal birth weight. Pyrimidine metabolism, pentose, glucuronate pathways were altered in macrosomia. [6, 8-10] However, the AA and AC metabolism in macrosomia is still unclear.

In the present study, LC-MS-based metabolomics approach was used to quantified the metabolic alteration in second trimester. We found the characteristic of metabolomics combined with clinical information and detected the potential risk factors of GDM. Moreover, we established a prognostic nomogram incorporating $\mathrm{AA}$ and $\mathrm{AC}$ for predicting macrosomia. Our finding provides a new insight that metabolic risk factors play a crucial role in GDM and associated macrosomia.

\section{Method}

\section{Subject}

This study is an observational retrospective study. Between June 2017 and April 2018,a total of 450 pregnant women in the second-trimester participated this study at Women's Hospital, school of medicine, Zhejiang University in Hangzhou. These women were blind, random chosen. The study design has been approved by the Ethics Committee of the hospital. All participants were of Chinese Han ethnicity. We obtain maternal characteristics information as follow: 
gravidity, parity, age, height, pre-pregnancy BMI, weight gain during pregnancy, using assisted reproductive technique (ART), method of pregnancy termination. The follow information of the offsprings were collected: termination of pregnancy weeks, sex, birth length, birth weight, head circumference, abdomen circumference and abdomen minus head circumference (data from fetal growth measurement ultrasound before labor).

GDM was defined according to the Chinese Current Care Guidelines for GDM as one or more pathological glucose values in a standard oral glucose tolerance test (OGTT). [11] The diagnostic thresholds were: fasting plasma glucose: $5.1 \mathrm{mmol} / \mathrm{L}, 1 \mathrm{H} 10.0 \mathrm{mmol} / \mathrm{L}$, and $2 \mathrm{H} 8.5 \mathrm{mmol} / \mathrm{L}$.[12] According to the guideline, 56 GMD cases were diagnosed. We randomly sampled 112 women without GDM from residual pregnant women participated in this study, frequency-matched to cases on birth day, age, delivery mode, and number of fetuses with 2:1 ratio. The common definition of macrosomia is birth weight over $4000 \mathrm{~g}$.

\section{Metabolic profiling detection by LC-MS/MS}

We aim to investigate the plasma AA and AC level of pregnant women at the second-trimester. We also obtain their neonate blood plasma sample. The blood samples were stored $-20^{\circ} \mathrm{C}$. [13] Furthermore, sample preparation used in tandem mass spectrometry (4000 QTrapTM; AB Sciex, Darmstadt, Germany) testing the concentration. The method used in the present study was essentially a modification of the procedure described elsewhere. [14] AA and AC were quantified using appropriate isotope-labelled standards. LC separation was performed on an Acquity UPLC HSS T3 column $\left(2.1^{\star} 100 \mathrm{~mm}, 100 \mathrm{~A}^{\circ}\right.$, $1.8 \mathrm{Im}$ particle size; Waters Corporation, MA) using water with $0.1 \%$ formic acid, $5 \mathrm{mM}$ ammonium acetate and $0.015 \%$ heptafluorobutyric acid as solvent $\mathrm{A}$ and methanol with $0.1 \%$ formic acid and 5Mm detected with a Xevo-G2-QTOF MS (Waters Corp.) operating in positive mode. Raw data were processed using Targetlynxas described previously. Accuracy of quantification was below $6 \%$ for all quantified metabolites except glutamic acid (13.9\%). Quantitative data were obtained using MetIDQTM Software. [15]

\section{Statistical analysis}

Data in figures and data were shown as mean $\pm S D$. Statistical methods Evidence for a difference between the two groups was tested by Student's t-test. Oneway ANOVA was used in more than two groups. For correlation analysis, the Spearman correlation coefficient was calculated in the level of AA and AC between the second trimester pregnancy adults and their neonates. In PLS-DA, metabolomics data were log transformed to ensure a normally distributed data set, R2 (goodness of fitness) and Q2 (goodness of prediction) were assessed in the PLS-DA models. In the univariate analyses, A Mann-Whitney U test was used to compare the variables GDM and control group. The values of serum AA and AC were log-transformed and standardized before entered into a logistic regression model. Odds ratios (ORs) and $95 \%$ confidence intervals (Cls) are reported per standard deviation. All statistical analysis was performed using IBM SPSS 23.0 edition, SIMCA 14.0, R vision 3.6.0. A significance level of 0.05 was used for all statistical tests.

\section{Results}

\section{Comparison of stages of pregnancy}

Baseline characteristics of 450 study participants divided into different categories according to natural conditions of pregnant women (gravidity, parity, age, height, pre-pregnancy BMI, weight gain, ART, method of pregnancy termination). The statistically significant results are shown in Table 1. In age subgroup, there was a trend that the level of Ala, Met, BCAA囚leucine, isoleucine, valine), AA (phenylalanine, tyrosine) ,several acylcarnitines (C2,C3, C4DC+C5OH, C16, $\mathrm{C} 18, \mathrm{C} 18: 1$ ) were higher in age $>35$ group, whereas the level of $\mathrm{CO}$ (free carnitine), Gly in age $>35$ group was lower. In pre-pregnancy $\mathrm{BMI} \geq 25.0$ and 30 subgroup, ALA, BCAA囚leucine, isoleucine, valine), AAA (phenylalanine, tyrosine), several acylcarnitines (C2 C3 C5 C16 C18:1) were higher. These metabolites were increased with pre-pregnancy BMI. In weight gain group, BCAA囚leucine, isoleucine, valine) and AAA(phenylalanine, tyrosine), C3, C5, C16, C18:1 were higher in weight gain $\geq 20 \mathrm{~kg}$ group than other group, while C0 was lower. In ART group, Ala, Arg C2, C3, C5 were higher while C0, Gly were lower. Our clinical characteristics stratified that the plasma level of AA and AC (contain free carnitine) showed no statistical difference in gravity, parity, height, method of pregnancy termination subgroup.

\section{Comparison of newborns}

Additionally, we investigated the maternal level of AA and AC in second-trimester pregnancy under the different neonate subgroup \termination of pregnancy weeks, sex, birth length, birthweight, head circumference, abdomen circumference and abdomen minus head circumference区. The result was shown in table 2 . In birth weight $>4000 \mathrm{~g}$ group, we found Ala, Arg, BCAA and AAA and several acylcarnitines (C2, C3, C5, C16, C18:1) were higher, these metabolites increased with birth weight, while $\mathrm{C} 0$ was lower. In abdomen circumference $>35 \mathrm{~cm}$ group, Ala, BCAA and AAA and several acylcarnitines (C2, C3, C5, C16) were higher, C0 and Gly were lower. In abdomen minus circumference subgroup, the metabolites characters have the similar trend with abdomen circumference $>35 \mathrm{~cm}$ subgroup. AA and AC have no significant difference in other subgroups.

\section{Correlation between maternal and newbom in amino acids and carnitine.}

We calculated the correlation between the corresponding values of the carnitine profile of the mothers at second trimester and their own newborns. Significant positive correlations were seen for leu, $\mathrm{Val}, \mathrm{Phe}, \mathrm{C}, \mathrm{C} 2, \mathrm{C} 3, \mathrm{C} 4 \mathrm{DC}+\mathrm{C} 5 \mathrm{OH}, \mathrm{C} 16, \mathrm{C} 18: 1$ (Table 3). Other AA and AC have no statistic significantly, these were not shown in table. We found the level of carnitine in neonate is less than adult.

\section{Different metabolite distribution in incorporated group}

We examined the serum metabolite in GDM patients and control. Our study used PLS-DA model (R2=0.527, Q2 $=0.464)$ to analyze difference between two groups. The PLS-DA scatterplot showed a clear class separation with GDM at the left ang the control group at the right. Furthermore, we used variable importance for the projection (VIP) to estimate the contribution of every metabolite to class separation (GDM vs. control). A VIP value >1 were considered with 
a high contribution to class separation. The VIP analysis showed that C0 plays a main role in class separation, followed by LEU+ILE+PRO-OH, C3, Phe, C18, TYR, C16, C2, GLY, ALA, VAL, C4DC+C5OH, C6DC, C8, C18:1.

\section{Cluster correlation heat map}

The heatmap (Fig. 1) depicting the inter correlations among metabolites revealed two predominant clusters of intercorrelated metabolites: AC (C2, C4, C6 and C8) and AA (Ala, Met, Phe, Leu+ILE+PRO-OH, and Val). The five metabolites included in the final model are showed strongly inter correlation. They are not strongly intercorrelated with each other, thereby avoiding problems of multi-collinearity in the regression model.

\section{Clinical characteristics of GDM patients, and compare AA and AC level with control}

Table 4 showed the characteristics of 56 GDM patients and 112 matched patients without GDM. As shown, there was insignificant difference in maternal age, height, pre pregnancy weight, gestational week at delivery, nulliparous between two groups. By contrast, pre-pregnancy BMI, weight gain, the number of assisted reproduction technology, the serum Ala, LEU+ILE+PRO-OH, Phe, TYR, Val, C2, C3, C4DC+C5OH, C6DC, C8, C16, C18, C18:1 were higher, while C0, $\mathrm{CO} /$ acylcarnitine were lower in GDM group. We selected significant factors from univariate analysis to enter multivariate analysis to examine whether act independently. Pre-pregnancy BMI (OR=1.15 per SD, 95\% Cl=1.06-1.78), weight gain (OR= 1.18, 95\% Cl= 1.03-1.64), LEU+ILE+PRO-OH (OR=1.31, 95\%Cl=1.171.23), $\operatorname{TYR}(\mathrm{OR}=1.34,95 \% \mathrm{Cl}=1.09-3.30), \mathrm{C}$ /acylcartine $(\mathrm{OR}=0.82,95 \% \mathrm{Cl}=0.72-0.98), \mathrm{CO}(\mathrm{OR}=0.70,95 \% \mathrm{Cl}=0.60-0.83), \mathrm{C} 3(\mathrm{OR}=1.03,95 \% \mathrm{Cl}=1.02-2.08), \mathrm{C} 16$ $(\mathrm{OR}=1.30,95 \% \mathrm{Cl}=1.12-4.28), \mathrm{C} 18(\mathrm{OR}=\cdot 1.2795 \% \mathrm{Cl}=1.00-3.27)$ were statistically associated with $\mathrm{GDM}$. These factors can work as independent risk factors involve in the process of GDM.

\section{A nomogram for predicting macrosomia}

Macrosomia is relatively associated with GDM. Here, we investigated the clinical characteristics, AA and AC metabolite between GDM with macrosomia and GDM without macrosomia (Table 5 and Figure 2). We found pre-pregnancy weight, BMI, weight gain, LEU+ILE+PRO-OH, TYR, Val, C0, C2, C3, C16, C18 were higher $(P<0.05)$. In multivariate analysis, we found pre-pregnancy BMI, weight gain, C0, C3, C16, LEU, TYR were evaluated $(P<0.05)$. These factors are independent risk factors involve in the process of GDM-induced macrosomia. The nomogram predicting GDM induced macrosomia incorporated these significant variables pre-pregnancy BMI, weight gain, C0, C3, C16, LEU, TYR. Among these metabolites, C0 deficiency showed highest OR (OR=0.759, 95\%Cl= 0.50-0.87). Vertical lines should be drawn from the correct location from each prognostic factor. "Total points" which could be obtained by add all points axis to the bottom axes to make the conversion into a macrosomia probability.

\section{Discussion}

In summary, we have identified metabolic alteration in the second trimester. We found 15 metabolites significantly related to GDM by using PLS-DA approach. Combined these metabolites with clinical information, multivariable logistic regression demonstrated that pre-pregnancy BMI, weight gain, LEU + ILE + PRO-OH, TYR, C0/acylcarnitine, C0, C3, C16, C18 are independent metabolic risk factors associated with GDM. At last, we developed a nomogram predicting probability of macrosomia based on carnitine related metabolites. Metabolomics is increasingly used in GDM capturing disease-relevant metabolic changes. To our knowledge, this is the first study report metabolic alteration during the second trimester pregnancy and metabolic risk factors in GDM during the second trimester based on carnitine related metabolomics and use nomogram predicting macrosomia.

Several experimental works have indicated characters of carnitine related metabolites during pregnancy. $[8,16,17]$ Total carnitine consisted of free carnitine and acylcarnitine. Free carnitine is required for fatty acid transferring into mitochondrial membrane, the ester carnitines are formed in the process. Ester carnitine presented as acylcarnitine was released into plasma. Fatty acid $\beta$-oxidation is associated uniquely with energy maintenance for maternal and neonate. [18] If fatty acid $\beta$-oxidation dysfunction, fetal growth and development will occur serious damage, such as macrosomia, preterm, neonatal seizure. Previous studies reported, the level of free carnitine is low not due to more carnitine in ester form. (16-18) An interesting finding appears there is a pronounced fall of the plasma content of free carnitine, acylcarnitine, total carnitine during pregnancy. [19] In our study, we found AAA (Tyr, Phe), BCAA (leu, Isoleu, val), other amino acids (Met, Ala, Arg) and some acylcarnitine (CO, C2, C3, C4DC + C5OH, C5, C16, C18, C18:1) are increased in Age > 35 group, BMI $\geq 30$, weight gain $>20 \mathrm{~kg}$ group. However, $\mathrm{CO}$ and Gly were decreased. Our study result is consistent with other literature on maternal metabolites associated with $\mathrm{BMI}$, glucose level, insulin sensitivity. Different ancestry groups have in common and independent aspects affect metabolites, induced diverse pregnancy outcomes. [20] Therefore, metabolomic profiling studies need to found China discipline.

GDM is defined as glucose intolerance dysfunction during pregnancy. The prevalence of GDM is increased from 5\% up to 14\% in USA. [21] GDM has adverse effects on both maternal and neonatal health. Identification metabolomics risk factor of GDM is critical to medical diagnosis and to minimize the negative consequence of GDM mother and offspring. [22] GDM has pathophysiological to T2DM. The metabolite profiles in GDM were considered to be similar with T2DM. [23] Carnitine, BCAA, AAA, FFAs were confirmed a strong relationship with diabetes. [24] In Chen's cohort study the evaluated serum level of five AAs were closely associated with people who were diagnosed with diabetes 10 years later. [6] Wang's study reported BCAA and AAA level progressively upraised in obese, hyperlipemia, high fructose diet and high fat diet group. [25] Valine acted as a critical role stand out with $251 \%$ increase in the GDM onset process. [6] Our study is consistent with these previous studies. In our study, the class separation of GDM and normal control was determined by C0, LEU + ILE + PRO-OH, C3, Phe, C18, etc. However, conflict observation were reported metabolic markers in T2DM may not have the same predictive power in GDM, the BCAA did not differ significantly between GDM and control in the first trimester. [26] The discrepancies may arise from different stage involved in the studies. We think it more appropriate to directly metabolomics in the second trimester.

One model clarified there is a phenomenon obese GDM patients compensate $\beta$ - oxidation increased. This process accelerates catabolism of fatty acids, resulting the acylcarnitine accumulation in mitochondria. Acylcarnitine was transported from mitochondria into plasma. The excess of acylcarnitine in 
mitochondria is harmful for metabolism dynamic balance due to aerobic glycolysis disturbed. Acylcarnitine C16:1, called hexadecenoy-L-carnitine, for determine step of transporting long-chain fatty acid into mitochondria. [27] Moreira's study investigated C16:1 and short-chain acylcarnitine increased with two weeks orange juice consumption. These results are in accordance with our observation. We hypothesize that the evaluated AC level may be an early maker and driver in aerobic glycolysis dysfunction. [28] In Battsetseg's study, they found C6 and C8 reached 1.5 fold increased in mice with pancreatic $\beta$-cell dysfunction.[17] In our study, we did not find the medium-chain acylcarnitine difference with GDM and normal control. Our Medium-Chain Acylcarnitine data is relatively low in absolute value, and it is difficult to draw a conclusion that the difference is statistically significant. Medium-Chain Acylcarnitine have higher metabolic efficiency and accumulates into short-chain acylcarnitine. [29] A number of studies have reported the association between AC and insulin resistance. Accumulated $A C$ impaired $\beta$ cell function and reduced insulin synthesis. [17] Another AC maybe impaired signaling through mammalian target of rapamycin uncoupling downstream signal transduction of insulin as one possible mechanism. [30] L-carnitine is beneficial for lowering lipid level by activating fatty acid fatty oxidation reducing the accumulation of $\mathrm{AC}$ and also effective in insulin sensitivity.

Birth weight plays an important role in neonate. It can represent newborn's nutrition and development status. Macrosomia is characterized by asymmetric growth of abdominal circumference and fat accumulation. [31] Pedersen's hypothesis could explain the pathophysiology of macrosomia. Maternal hyperglycemia can lead to fetal hyperinsulinemia status resulting in glucose utilization increasing. [32] When maternal glycemic not under control, the accumulated maternal serum glucose can cross the placenta. [33] Fetal synthesizes adipose tissue in abdomen. Lipid metabolism is another vital factor affecting fetal growth in uterus. Previous studies conducted high TG, high LDL, and low HDL were independently associated with macrosomia and low large for gestational age. A survey has found uracil and 2-methylfumarate are risk factors and elaidic acid, 4-aminobutyric acid are protective factors in macrosomia. [10] Our study found C0 is a protective factor in macrosomia. C3, C16, LEU, TYR are risk factors in macrosomia. Therefore, the characterization of metabolism may enable us to discover reliable biomarkers and enable us to set up a nomogram model to predict macrosomia.

Carnitine metabolite was investigated in assisted reproduction, obstetrics, pediatrics and other fields. Some papers reported administration L-carnitine induced the production of PGE1 and PGE2 by macrophages impairs fertility in female mice, [34] but oral L-carnitine may be beneficial for total sperm concentration, sperm total motility, sperm morphology.[35] Serum acylcarnitine metabolomics in first trimester were used to improve the accuracy of preeclampsia diagnosis. [36] In fetal, carnitine was involved in the production of pulmonary surfactant. [37]

Our study first found the AA and AC metabolic characteristics in second trimester pregnancy, the significant difference in the GDM metabolites and independent risk factor affecting GDM. Furthermore, we induced a model to predict macrosomia. We speculated carnitine related metabolomics may be helpful to investigate GDM etiology and prophylactic treatment in GDM induced macrosomia. Understanding metabolic-related signaling pathways can identify novel therapeutic targets. Early pregnancy is a critical period of organ development, which is easily affected by external influences and causes malformation.[38] In the third trimester, it is too late to intervene. [39] The second trimester is considered a safe phase and some intervention given, such as oral L-carnitine, to reduce the incidence of GDM. Oral L-carnitine supplementation induce some side effects, more intimated researches are needed. [40] Our study has several limitations. We should expand the sample size and detect more metabolites to understand the correlation between various metabolites. Our study was a retrospective case control study and just finding GDM risk factor, the real metabolomics value need cohort to confirm.

Our observations have important significance in public health and clinical implications. We confirmed the difference metabolites in GDM. These may participate in the process of pathological formation. We can develop targeted therapies of these metabolic patterns in future. The macrosomia model can estimate birthweight more accurate. This will guide the termination of pregnancy, avoid the risk of shoulder dystocia, and reduce the rate of cesarean section. We will give interventions in the second trimester to reduce the incidence of pregnancy complications.

\section{Conclusion}

In conclusion, by analyzing the metabolic alteration in the second trimester, we discovered the metabolic profiling in different subgroups according to clinical information. We obtained the metabolic trend is similar with maternal result in fetal clinical information. The major novel finding of this study was that prepregnancy BMI, weight gain, LEU + ILE + PRO-OH, TYR, C0/acylcarnitine, C0, C3, C16, C18 are independent risk factors associated with GDM. Pre-pregnancy $\mathrm{BMI}$, weight gain, C0, C3, C16, LEU, TYR are independent risk factors involve in the process of GDM-induced macrosomia. Based on these metabolic factors and clinical information, a nomogram was conducted to predict the macrosomia. Our findings from this research serve as a reminder in the future to study and help understanding the underlying biochemical pathology of GDM and reduce the incidence of GDM.

\section{Abbreviations}

GDM: gestational diabetes mellitus; AA: amino acid; BCAA: branched-chain amino acid; AAA: aromatic amino acid; AC: acetylcarnitine; OGTT: oral glucose tolerance test; ART: assisted reproductive technique; OR: Odds ratios; $\mathrm{Cl}$ : confidence intervals

\section{Declarations}

\section{Acknowledgements}

We thank all patients for their participation.

\section{Authors' contributions}

Qiong Luo and Man Sun conceived and designed the experiments. Binqiao Wang and Yunping Ding collected the data. Ruopeng Weng and Sainan He analyzed and interpreted the data. Man Sun and Baihui zhao wrote the paper. All authors reviewed and approved the paper. 
Funding

This research was supported by Natural Science Foundation of Zhejiang province, China (Grant No. LQ20H040008هLY20H040009).

Availability of data and materials

The datasets during and/or analyzed during the current study available from the corresponding author on reasonable request. Ethics approval and consent to participate

This study was approved by the Ethics Committee of Women's Hospital, School of Medicine, Zhejiang University. All study participants were included in the study after giving written informed consent.

\section{Consent for publication}

Not applicable.

Competing interests

The authors declare no conflicts of interest.

Author details

Department of Obstetrics, Women's Hospital, School of Medicine, Zhejiang University.

No.1, Xueshi Road, Shangchen District, Hangzhou, China.

\section{References}

1. Vrijkotte TG, Krukziener N, Hutten BA, Vollebregt KC, van Eijsden M, Twickler MB: Maternal lipid profile during early pregnancy and pregnancy complications and outcomes: the ABCD study. J Clin Endocrinol Metab 2012, 97:3917-3925.

2. Bianco ME, Josefson JL: Hyperglycemia During Pregnancy and Long-Term Offspring Outcomes. Curr Diab Rep 2019, 19:143.

3. Hoppel C: The role of carnitine in normal and altered fatty acid metabolism. Am J Kidney Dis 2003, 41:S4-12.

4. Chamberlain JJ, Doyle-Delgado K, Peterson L, Skolnik N: Diabetes Technology: Review of the 2019 American Diabetes Association Standards of Medical Care in Diabetes. Ann Intern Med 2019.

5. Geach T: Diabetes: A metabolomic signature to predict the transition from GDM to T2DM. Nat Rev Endocrino/ 2016, 12:498.

6. Chen T, Ni Y, Ma X, Bao Y, Liu J, Huang F, Hu C, Xie G, Zhao A, Jia W, Jia W: Branched-chain and aromatic amino acid profiles and diabetes risk in Chinese populations. Sci Rep 2016, 6:20594.

7. Simcox J, Geoghegan G, Maschek JA, Bensard CL, Pasquali M, Miao R, Lee S, Jiang L, Huck I, Kershaw EE, et al: Global Analysis of Plasma Lipids Identifies Liver-Derived Acylcarnitines as a Fuel Source for Brown Fat Thermogenesis. Cell Metab 2017, 26:509-522.e506.

8. Nevalainen J, Sairanen M, Appelblom H, Gissler M, Timonen S, Ryynanen M: First-Trimester Maternal Serum Amino Acids and Acylcarnitines Are Significant Predictors of Gestational Diabetes. Rev Diabet Stud 2016, 13:236-245.

9. Swank ML, Caughey AB, Farinelli CK, Main EK, Melsop KA, Gilbert WM, Chung JH: The impact of change in pregnancy body mass index on macrosomia. Obesity (Silver Spring) 2014, 22:1997-2002.

10. Sun H, Wang YC, Wang CC, Xu XX, Wang YH, Yan HT, Yang XJ: Metabolic profiling of umbilical cord blood in macrosomia. Int J Obes (Lond) 2018, 42:679685.

11. Kim C, Newton KM, Knopp RH: Gestational diabetes and the incidence of type 2 diabetes: a systematic review. Diabetes Care 2002, 25:1862-1868.

12. Bellamy L, Casas JP, Hingorani AD, Williams D: Type 2 diabetes mellitus after gestational diabetes: a systematic review and meta-analysis. Lancet 2009 , 373:1773-1779

13. Peng M, Liu L, Jiang M, Liang C, Zhao X, Cai Y, Sheng H, Ou Z, Luo H: Measurement of free carnitine and acylcarnitines in plasma by HILIC-ESI-MS/MS without derivatization. J Chromatogr B Analyt Technol Biomed Life Sci 2013, 932:12-18.

14. Bene J, Komlosi K, Havasi V, Talian G, Gasztonyi B, Horvath K, Mozsik G, Hunyady B, Melegh B, Figler M: Changes of plasma fasting carnitine ester profile in patients with ulcerative colitis. World J Gastroentero/ 2006, 12:110-113.

15. Jia C, Xu H, Xu Y, Xu Y, Shi Q: Serum metabolomics analysis of patients with polycystic ovary syndrome by mass spectrometry. Mol Reprod Dev 2019, 86:292-297.

16. Talian GC, Komlosi K, Decsi T, Koletzko B, Melegh B: Determination of carnitine ester patterns during the second half of pregnancy, at delivery, and in neonatal cord blood by tandem mass spectrometry: complex and dynamic involvement of carnitine in the intermediary metabolism. Pediatr Res 2007 , 62:88-92.

17. Batchuluun B, Al Rijjal D, Prentice KJ, Eversley JA, Burdett E, Mohan H, Bhattacharjee A, Gunderson EP, Liu Y, Wheeler MB: Elevated Medium-Chain Acylcarnitines Are Associated With Gestational Diabetes Mellitus and Early Progression to Type 2 Diabetes and Induce Pancreatic beta-Cell Dysfunction. Diabetes 2018, 67:885-897. 
18. Natarajan SK, Ibdah JA: Role of 3-Hydroxy Fatty Acid-Induced Hepatic Lipotoxicity in Acute Fatty Liver of Pregnancy. Int J Mol Sci $2018,19$.

19. Lindsay KL, Hellmuth C, Uhl O, Buss C, Wadhwa PD, Koletzko B, Entringer S: Longitudinal Metabolomic Profiling of Amino Acids and Lipids across Healthy Pregnancy. PLoS One 2015, 10:e0145794.

20. Jacob S, Nodzenski M, Reisetter AC, Bain JR, Muehlbauer MJ, Stevens RD, Ilkayeva OR, Lowe LP, Metzger BE, Newgard CB, et al: Targeted Metabolomics Demonstrates Distinct and Overlapping Maternal Metabolites Associated With BMI, Glucose, and Insulin Sensitivity During Pregnancy Across Four Ancestry Groups. Diabetes Care 2017, 40:911-919.

21. Metzger BE, Lowe LP, Dyer AR, Trimble ER, Chaovarindr U, Coustan DR, Hadden DR, McCance DR, Hod M, Mclntyre HD, et al: Hyperglycemia and adverse pregnancy outcomes. N Engl J Med 2008, 358:1991-2002.

22. Lowe WL, Scholtens DM, Kuang A, Linder B, Lawrence JM, Lebenthal Y, McCance D, Hamilton J, Nodzenski M, Talbot O, et al: Hyperglycemia and Adverse Pregnancy Outcome Follow-up Study (HAPO FUS): Maternal Gestational Diabetes Mellitus and Childhood Glucose Metabolism. Diabetes Care 2019, 42:372-380.

23. Dessi A, Marincola FC, Fanos V: Metabolomics and the great obstetrical syndromes-GDM, PET, and IUGR. Best Pract Res Clin Obstet Gynaecol 2015, 29:156-164.

24. Strand E, Rebnord EW, Flygel MR, Lysne V, Svingen GFT, Tell GS, Loland KH, Berge RK, Svardal A, Nygard O, Pedersen ER: Serum Carnitine Metabolites and Incident Type 2 Diabetes Mellitus in Patients With Suspected Stable Angina Pectoris. J Clin Endocrinol Metab 2018, 103:1033-1041.

25. Wang SM, Yang RY, Wang M, Ji FS, Li HX, Tang YM, Chen WX, Dong J: Identification of serum metabolites associated with obesity and traditional risk factors for metabolic disease in Chinese adults. Nutr Metab Cardiovasc Dis 2018, 28:112-118.

26. Roy C, Tremblay PY, Anassour-Laouan-Sidi E, Lucas M, Forest JC, Giguere Y, Ayotte P: Risk of gestational diabetes mellitus in relation to plasma concentrations of amino acids and acylcarnitines: A nested case-control study. Diabetes Res Clin Pract 2018, 140:183-190.

27. Senyilmaz-Tiebe D, Pfaff DH, Virtue S, Schwarz KV, Fleming T, Altamura S, Muckenthaler MU, Okun JG, Vidal-Puig A, Nawroth P, Teleman AA: Dietary stearic acid regulates mitochondria in vivo in humans. Nature communications 2018, 9:3129-3129.

28. Moreira V, Brasili E, Fiamoncini J, Marini F, Miccheli A, Daniel H, Lee JJH, Hassimotto NMA, Lajolo FM: Orange juice affects acylcarnitine metabolism in healthy volunteers as revealed by a mass-spectrometry based metabolomics approach. Food Res Int 2018, 107:346-352.

29. Seo W-K, Jo G, Shin M-J, Oh K: Medium-Chain Acylcarnitines Are Associated With Cardioembolic Stroke and Stroke Recurrence. Arteriosclerosis, thrombosis, and vascular biology 2018, 38:2245-2253.

30. Wildberg C, Masuch A, Budde K, Kastenmüller G, Artati A, Rathmann W, Adamski J, Kocher T, Völzke H, Nauck M, et al: Plasma Metabolomics to Identify and Stratify Patients With Impaired Glucose Tolerance. The Journal of clinical endocrinology and metabolism 2019, 104:6357-6370.

31. Billionnet C, Mitanchez D, Weill A, Nizard J, Alla F, Hartemann A, Jacqueminet S: Gestational diabetes and adverse perinatal outcomes from 716,152 births in France in 2012. Diabetologia 2017, 60:636-644.

32. Catalano PM, Hauguel-De Mouzon S: Is it time to revisit the Pedersen hypothesis in the face of the obesity epidemic? Am J Obstet Gynecol 2011, 204:479487.

33. Kc K, Shakya S, Zhang H: Gestational diabetes mellitus and macrosomia: a literature review. Ann Nutr Metab 2015, 66 Suppl 2:14-20.

34. Kyvelidou C, Sotiriou D, Antonopoulou T, Tsagkaraki M, Tserevelakis GJ, Filippidis G, Athanassakis I: I-Carnitine affects preimplantation embryo development toward infertility in mice. Reproduction 2016, 152:283-291.

35. Salas-Huetos A, Rosique-Esteban N, Becerra-Tomás N, Vizmanos B, Bulló M, Salas-Salvadó J: The Effect of Nutrients and Dietary Supplements on Sperm Quality Parameters: A Systematic Review and Meta-Analysis of Randomized Clinical Trials. Advances in Nutrition 2018, 9:833-848.

36. Koster MPH, Vreeken RJ, Harms AC, Dane AD, Kuc S, Schielen PCJI, Hankemeier T, Berger R, Visser GHA, Pennings JLA: First-Trimester Serum Acylcarnitine Levels to Predict Preeclampsia: A Metabolomics Approach. Disease Markers 2015, 2015:1-8.

37. Kepka A, Chojnowska S, Okungbowa OE, Zwierz K: The role of carnitine in the perinatal period. Dev Period Med 2014, 18:417-425.

38. Kwak-Kim J, Bao S, Lee SK, Kim JW, Gilman-Sachs A: Immunological modes of pregnancy loss: inflammation, immune effectors, and stress. American journal of reproductive immunology (New York, NY: 1989) 2014, 72:129-140.

39. Bick D: The challenge of obesity during pregnancy: When to intervene and what could work? Midwifery 2015, 31:655-656.

40. Koeth RA, Lam-Galvez BR, Kirsop J, Wang Z, Levison BS, Gu X, Copeland MF, Bartlett D, Cody DB, Dai HJ, et al: I-Carnitine in omnivorous diets induces an atherogenic gut microbial pathway in humans. The Journal of clinical investigation 2019, 129:373-387.

\section{Tables}

Table 1 the plasma AA and AC level of the second trimester in subgroup according to pregnant clinical characteristic 


\begin{tabular}{|c|c|c|c|c|c|c|c|c|c|c|c|c|c|}
\hline & \multicolumn{3}{|c|}{ age } & \multirow[t]{2}{*}{$P$-value } & \multicolumn{4}{|c|}{ pre-pregnancy BMI } & \multirow{2}{*}{$\begin{array}{c}P- \\
\text { value }\end{array}$} & \multicolumn{4}{|c|}{ weight gain $\llbracket \mathrm{kg} \rrbracket$} \\
\hline & $\square 25$ & $25-35$ & $\square 35$ & & $\square 18.5$ & 18.5-24.9 & $25.0-29.9$ & $\geq 30$ & & $\square 12.5$ & $12.5-15$ & $15-20$ & $\square 20$ \\
\hline $\mathrm{n}$ & 12 & 247.00 & 191.00 & & 9 & 253 & 153 & 35 & & 127 & 117 & 150 & 56 \\
\hline ALA & $313.49 \pm 42.47$ & $336.71 \pm 64.23$ & $350.47 \pm 69.38$ & $0.039^{*}$ & $323.49 \pm 29.63$ & $322.42 \pm 67.37$ & $323.21 \pm 65.72$ & $323.51 \pm 62.88$ & 0.410 & $328.49 \pm 26.56$ & $330.43 \pm 64.54$ & $322.13 \pm 65.61$ & $328.52 \pm 60.58$ \\
\hline ARG & $9.04 \pm 4.56$ & $8.64 \pm 5.71$ & $8.54 \pm 3.2$ & 0.350 & $8.63 \pm 5.62$ & $8.54 \pm 4.18$ & $9.21 \pm 5.71$ & $8.53 \pm 3.42$ & 0.613 & $7.63 \pm 5.34$ & $7.48 \pm 3.96$ & $7.31 \pm 5.13$ & $7.68 \pm 3.68$ \\
\hline GLY & $228.41 \pm 31.83$ & $223.05 \pm 41.31$ & $218.51 \pm 39.66$ & $0.027 *$ & $219.31 \pm 29.56$ & $220.02 \pm 49.47$ & $222.82 \pm 36.77$ & $223.60 \pm 40.51$ & 0.080 & $223.34 \pm 29.87$ & $223.93 \pm 50.47$ & $222.82 \pm 36.77$ & $228.60 \pm 40.51$ \\
\hline $\begin{array}{l}\text { LEU+ILE+PRO- } \\
\mathrm{OH}\end{array}$ & $127.92 \pm 30.24$ & $130.56 \pm 30.02$ & $139.55 \pm 25.34$ & $0.010^{*}$ & $124.66 \pm 31.03$ & $127.18 \pm 28.34$ & $131.46 \pm 30.57$ & $134.58 \pm 19.77$ & $0.013^{*}$ & $121.78 \pm 31.04$ & $124.87 \pm 28.34$ & $126.98 \pm 30.58$ & $129.58 \pm 19.78$ \\
\hline MET & $9.67 \pm 1.95$ & $10.66 \pm 2.92$ & $11.55 \pm 2.26$ & $0.024^{*}$ & $9.77 \pm 2.19$ & $9.54 \pm 2.65$ & $9.67 \pm 2.88$ & $9.75 \pm 2.03$ & 0.210 & $10.63 \pm 2.19$ & $10.77 \pm 2.65$ & $10.64 \pm 2.87$ & $10.85 \pm 2.03$ \\
\hline PHE & $47.55 \pm 8.83$ & $48.15 \pm 9.81$ & $49.56 \pm 8.07$ & $0.019^{*}$ & $44.78 \pm 8.14$ & $46.75 \pm 8.76$ & $48.72 \pm 10.07$ & $50.19 \pm 6.67$ & $0.039^{*}$ & $44.78 \pm 8.14$ & $46.75 \pm 8.96$ & $48.72 \pm 10.17$ & $49.20 \pm 6.67$ \\
\hline TYR & $37.17 \pm 7.62$ & $39.51 \pm 8.21$ & $42.51 \pm 8.8$ & $0.036^{*}$ & $36.61 \pm 8.59$ & $38.39 \pm 8.63$ & $39.98 \pm 8.07$ & $42.17 \pm 4.68$ & $0.031^{*}$ & $37.61 \pm 8.29$ & $38.39 \pm 8.33$ & $39.98 \pm 8.37$ & $40.17 \pm 4.68$ \\
\hline VAL & $133.89 \pm 34.58$ & $120.96 \pm 24.82$ & $118.61 \pm 25.75$ & $0.027 *$ & $103.61 \pm 28.57$ & $111.19 \pm 27.97$ & $116.49 \pm 24.51$ & $123.86 \pm 18.7$ & $0.045^{*}$ & $113.67 \pm 28.57$ & $119.19 \pm 27.91$ & $121.49 \pm 24.51$ & $123.86 \pm 18.7$ \\
\hline $\mathrm{C} 0$ & $20.26 \pm 3.86$ & $19.34 \pm 3.81$ & $16.18 \pm 7.55$ & $0.035^{*}$ & $19.15 \pm 3.8$ & $17.63 \pm 5.49$ & $16.43 \pm 4.76$ & $15.52 \pm 3.13$ & $0.038^{*}$ & $18.85 \pm 3.8$ & $17.83 \pm 5.45$ & $16.63 \pm 4.62$ & $15.28 \pm 3.14$ \\
\hline $\mathrm{C} 2$ & $2.08 \pm 0.54$ & $2.43 \pm 0.76$ & $2.61 \pm 0.92$ & $0.043^{*}$ & $1.84 \pm 0.49$ & $2.25 \pm 0.82$ & $2.48 \pm 0.78$ & $2.61 \pm 0.67$ & $0.017 *$ & $2.34 \pm 0.42$ & $2.49 \pm 0.56$ & $2.48 \pm 0.28$ & $2.39 \pm 0.67$ \\
\hline C3 & $0.52 \pm 0.14$ & $0.56 \pm 0.17$ & $0.58 \pm 0.2$ & $0.042^{*}$ & $0.42 \pm 0.14$ & $0.45 \pm 0.19$ & $0.48 \pm 0.18$ & $0.55 \pm 0.17$ & $0.015^{*}$ & $0.48 \pm 0.14$ & $0.55 \pm 0.19$ & $0.58 \pm 0.19$ & $0.65 \pm 0.17$ \\
\hline $\mathrm{C} 4 \mathrm{DC}+\mathrm{C} 5 \mathrm{OH}$ & $0.16 \pm 0.03$ & $0.14 \pm 0.05$ & $0.13 \pm 0.02$ & $0.014^{*}$ & $0.14 \pm 0.03$ & $0.14 \pm 0.06$ & $0.14 \pm 0.04$ & $0.14 \pm 0.04$ & 0.290 & $0.14 \pm 0.03$ & $0.14 \pm 0.06$ & $0.14 \pm 0.02$ & $0.14 \pm 0.04$ \\
\hline C5 & $0.05 \pm 0.02$ & $0.04 \pm 0.01$ & $0.04 \pm 0.01$ & 0.524 & $0.03 \pm 0.01$ & $0.04 \pm 0.02$ & $0.05 \pm 0.01$ & $0.06 \pm 0.01$ & $0.027 *$ & $0.02 \pm 0.01$ & $0.03 \pm 0.02$ & $0.04 \pm 0.01$ & $0.06 \pm 0.01$ \\
\hline C16 & $0.43 \pm 0.13$ & $0.44 \pm 0.17$ & $0.45 \pm 0.18$ & $0.019^{*}$ & $0.44 \pm 0.1$ & $0.47 \pm 0.19$ & $0.54 \pm 0.18$ & $0.68 \pm 0.28$ & $0.026^{*}$ & $0.42 \pm 0.15$ & $0.44 \pm 0.19$ & $0.45 \pm 0.18$ & $0.47 \pm 0.25$ \\
\hline C18 & $0.26 \pm 0.08$ & $0.27 \pm 0.11$ & $0.30 \pm 0.1$ & $0.032^{*}$ & $0.30 \pm 0.09$ & $0.28 \pm 0.13$ & $0.28 \pm 0.10$ & $0.29 \pm 0.17$ & 0.510 & $0.31 \pm 0.09$ & $0.31 \pm 0.13$ & $0.29 \pm 0.11$ & $0.30 \pm 0.17$ \\
\hline C18:1 & $0.48 \pm 0.13$ & $0.49 \pm 0.16$ & $0.54 \pm 0.24$ & $0.017^{*}$ & $0.32 \pm 0.12$ & $0.38 \pm 0.23$ & $0.46 \pm 0.19$ & $0.52 \pm 0.17$ & $0.019^{*}$ & $0.32 \pm 0.18$ & $0.41 \pm 0.26$ & $0.48 \pm 0.16$ & $0.55 \pm 0.16$ \\
\hline
\end{tabular}

Table 2 The maternal level of AA and AC in second-trimester pregnancy under the different neonate subgroup

\begin{tabular}{|c|c|c|c|c|c|c|c|c|c|c|c|c|c|}
\hline & \multicolumn{4}{|c|}{ birth weight } & \multirow{2}{*}{$\begin{array}{c}P- \\
\text { value }\end{array}$} & \multicolumn{3}{|c|}{ abdomen circumference } & \multirow{2}{*}{$\begin{array}{c}P- \\
\text { value }\end{array}$} & \multicolumn{3}{|c|}{ abdomen-head circumference $(\mathrm{cm})$} & \multirow{2}{*}{$\begin{array}{c}P- \\
\text { value }\end{array}$} \\
\hline & $<2800 \mathrm{~g}$ & $2800-3500 \mathrm{~g}$ & $3500-4000 \mathrm{~g}$ & $>4000 \mathrm{~g}$ & & $<32 \mathrm{~cm}$ & $32-35 \mathrm{~cm}$ & $>35 \mathrm{~cm}$ & & $<2$ & $2-5$ & 5 & \\
\hline$\overline{\text { ALA }}$ & $\begin{array}{c}329.73 \pm \\
47.57\end{array}$ & $\begin{array}{c}339.57 \pm \\
35.19\end{array}$ & $\begin{array}{c}346.52 \pm \\
32.87\end{array}$ & $\begin{array}{c}357.87 \pm \\
65.45\end{array}$ & $0.024^{*}$ & $\begin{array}{c}320.49 \pm \\
29.63\end{array}$ & $\begin{array}{c}326.68 \pm \\
23.67\end{array}$ & $\begin{array}{c}340.03 \pm \\
66.14\end{array}$ & $0.025^{*}$ & $\begin{array}{c}326.45 \pm \\
33.38\end{array}$ & $\begin{array}{c}339.90 \pm \\
35.95\end{array}$ & $\begin{array}{c}355.26 \pm \\
63.37\end{array}$ & $0.016^{*}$ \\
\hline ARG & $7.59 \pm 4.26$ & $8.25 \pm 5.76$ & $8.53 \pm 3.42$ & $8.60 \pm 5.16$ & $0.038^{*}$ & $6.63 \pm 5.62$ & $7.28 \pm 3.48$ & $\begin{array}{c}340.03 \pm \\
66.14\end{array}$ & 0.142 & $7.35 \pm 3.53$ & $9.07 \pm 5.68$ & $8.52 \pm 3.47$ & 0.022 \\
\hline GLY & $\begin{array}{c}223.19 \\
49.92\end{array}$ & $\begin{array}{c}222.73 \pm \\
36.98\end{array}$ & $\begin{array}{c}224.60 \pm \\
40.51\end{array}$ & $\underset{41.56}{223.42 \pm}$ & 0.635 & $\begin{array}{c}225.31 \\
29.56\end{array}$ & $\begin{array}{c}222.88 \\
50.48\end{array}$ & $\begin{array}{c}210.05 \\
36.52\end{array}$ & $0.017 *$ & $\begin{array}{c}228.68 \pm \\
50.26\end{array}$ & $\frac{221.91 \pm}{36.46}$ & $\begin{array}{c}218.61 \pm \\
41.17\end{array}$ & $0.024^{*}$ \\
\hline $\begin{array}{l}\text { LEU+ILE+PRO- } \\
\text { OH }\end{array}$ & $\begin{array}{c}127.22 \\
28.41\end{array}$ & $\begin{array}{c}131.27 \\
30.74\end{array}$ & $\begin{array}{c}132.58 \\
19.77\end{array}$ & $\underset{29.02}{135.11} \pm$ & $0.014^{*}$ & $\begin{array}{c}124.67 \\
31.03\end{array}$ & $\begin{array}{c}126.13 \pm \\
27.36\end{array}$ & $\begin{array}{c}130.56 \\
30.80\end{array}$ & $0.019 *$ & $\begin{array}{c}126.38 \\
27.34\end{array}$ & $\begin{array}{c}130.58 \pm \\
30.71\end{array}$ & $\begin{array}{c}132.62 \\
19.21\end{array}$ & $0.017 *$ \\
\hline PHE & $\begin{array}{c}46.74 \pm \\
8.93\end{array}$ & $\begin{array}{c}48.67 \pm \\
10.16\end{array}$ & $\begin{array}{c}49.19 \pm \\
6.67\end{array}$ & $\begin{array}{c}50.08 \\
9.45\end{array}$ & $0.028^{*}$ & $\begin{array}{c}46.77 \pm \\
8.14\end{array}$ & $\begin{array}{c}48.33 \pm \\
8.63\end{array}$ & $\begin{array}{c}49.39 \pm \\
10.18\end{array}$ & $0.033^{*}$ & $\begin{array}{c}46.38 \pm \\
8.60\end{array}$ & $\begin{array}{c}47.42 \pm \\
10.16\end{array}$ & $\underset{6.66}{48.99} \pm$ & $0.027 *$ \\
\hline TYR & $\begin{array}{c}38.23 \pm \\
8.63\end{array}$ & $\begin{array}{c}39.91 \\
8.13\end{array}$ & $\begin{array}{c}40.17 \pm \\
4.68\end{array}$ & $\begin{array}{c}42.42 \\
8.02\end{array}$ & $0.043^{*}$ & $\begin{array}{c}40.61 \\
8.59\end{array}$ & $\begin{array}{c}37.95 \pm \\
8.58\end{array}$ & $\begin{array}{c}39.73 \pm \\
8.17\end{array}$ & $0.241^{*}$ & $\begin{array}{c}37.99 \pm \\
8.55\end{array}$ & $\begin{array}{c}39.75 \pm \\
8.16\end{array}$ & $\begin{array}{c}40.95 \\
4.56\end{array}$ & $0.018^{*}$ \\
\hline VAL & $\begin{array}{c}118.73 \pm \\
27.77\end{array}$ & $\begin{array}{c}120.15 \\
24.61\end{array}$ & $\begin{array}{c}123.86 \pm \\
18.70\end{array}$ & $\frac{125.52}{25.15} \pm$ & $0.035^{*}$ & $\begin{array}{c}113.61 \\
28.57\end{array}$ & $\begin{array}{c}118.05 \\
27.70\end{array}$ & $\begin{array}{c}120.33 \\
24.09\end{array}$ & $0.026^{*}$ & $\begin{array}{c}118.04 \\
27.60\end{array}$ & $\begin{array}{c}120.25 \\
24.05\end{array}$ & $\begin{array}{c}123.30 \\
18.68\end{array}$ & $0.011^{*}$ \\
\hline $\mathrm{CO}$ & $\begin{array}{c}18.38 \pm \\
5.23\end{array}$ & $\begin{array}{c}17.37 \\
4.77\end{array}$ & $\begin{array}{c}16.52 \\
3.13\end{array}$ & $\begin{array}{c}15.40 \pm \\
4.74\end{array}$ & $0.026^{*}$ & $\begin{array}{c}18.15 \pm \\
3.80\end{array}$ & $\begin{array}{c}17.22 \\
5.18\end{array}$ & $\begin{array}{c}15.11 \\
4.04\end{array}$ & $0.018^{*}$ & $\begin{array}{c}19.19 \pm \\
5.16\end{array}$ & $\begin{array}{c}17.08 \pm \\
4.06\end{array}$ & $\begin{array}{c}15.61 \pm \\
3.14\end{array}$ & $0.014^{*}$ \\
\hline $\mathrm{C} 2$ & $2.30 \pm 0.82$ & $2.42 \pm 0.78$ & $2.49 \pm 0.67$ & $2.56 \pm 0.78$ & $0.023^{*}$ & $1.84 \pm 0.49$ & $2.55 \pm 0.82$ & $2.73 \pm 0.76$ & $0.023^{*}$ & $2.20 \pm 0.82$ & $2.47 \pm 0.76$ & $2.57 \pm 0.66$ & $0.033^{*}$ \\
\hline $\mathrm{C} 3$ & $0.46 \pm 0.18$ & $0.51 \pm 0.16$ & $0.53 \pm 0.17$ & $0.56 \pm 0.18$ & $0.014^{*}$ & $0.52 \pm 0.14$ & $0.54 \pm 0.16$ & $0.57 \pm 0.18$ & $0.024^{*}$ & $\begin{array}{c}0.44 \pm \\
0.164\end{array}$ & $0.51 \pm 0.18$ & $0.55 \pm 0.17$ & $0.013^{*}$ \\
\hline C5 & $0.02 \pm 0.01$ & $0.03 \pm 0.01$ & $0.04 \pm 0.01$ & $0.05 \pm 0.01$ & $0.037 *$ & $0.05 \pm 0.01$ & $0.04 \pm 0.01$ & $0.06 \pm 0.01$ & $0.025^{*}$ & $0.04 \pm 0.01$ & $0.05 \pm 0.01$ & $0.06 \pm 0.01$ & $0.026^{*}$ \\
\hline C16 & $0.42 \pm 0.12$ & $0.44 \pm 0.18$ & $0.47 \pm 0.20$ & $0.44 \pm 0.16$ & $0.027 *$ & $0.42 \pm 0.10$ & $0.43 \pm 0.12$ & $0.47 \pm 0.17$ & $0.026^{*}$ & $0.41 \pm 0.12$ & $0.44 \pm 0.18$ & $0.48 \pm 0.20$ & $0.015^{*}$ \\
\hline C18:1 & $0.48 \pm 0.15$ & $0.50 \pm 0.17$ & $0.52 \pm 0.17$ & $0.55 \pm 0.14$ & $0.017 *$ & $0.48 \pm 0.12$ & $0.49 \pm 0.15$ & $0.52 \pm 0.16$ & $0.027 *$ & $0.48 \pm 0.15$ & $0.49 \pm 0.16$ & $0.49 \pm 0.18$ & 0.123 \\
\hline
\end{tabular}

Table 3 correlation analysis of canitine profiles between mother in second trimester and neonate

\begin{tabular}{lcl}
\hline & Correlation coefficient & $P$ \\
\hline free canitine & 0.757 & 0.013 \\
\hline leu & 0.623 & 0.018 \\
\hline Val & 0.586 & 0.037 \\
Phe & 0.698 & 0.021 \\
\hline C2 & 0.656 & 0.032 \\
C3 & 0.713 & 0.018 \\
\hline C5 & 0.533 & 0.022 \\
C16 & 0.682 & 0.032 \\
C18:1 & 0.529 & 0.034 \\
\hline
\end{tabular}


Table 4: Selected characteristics of women with GDM and matched control women.

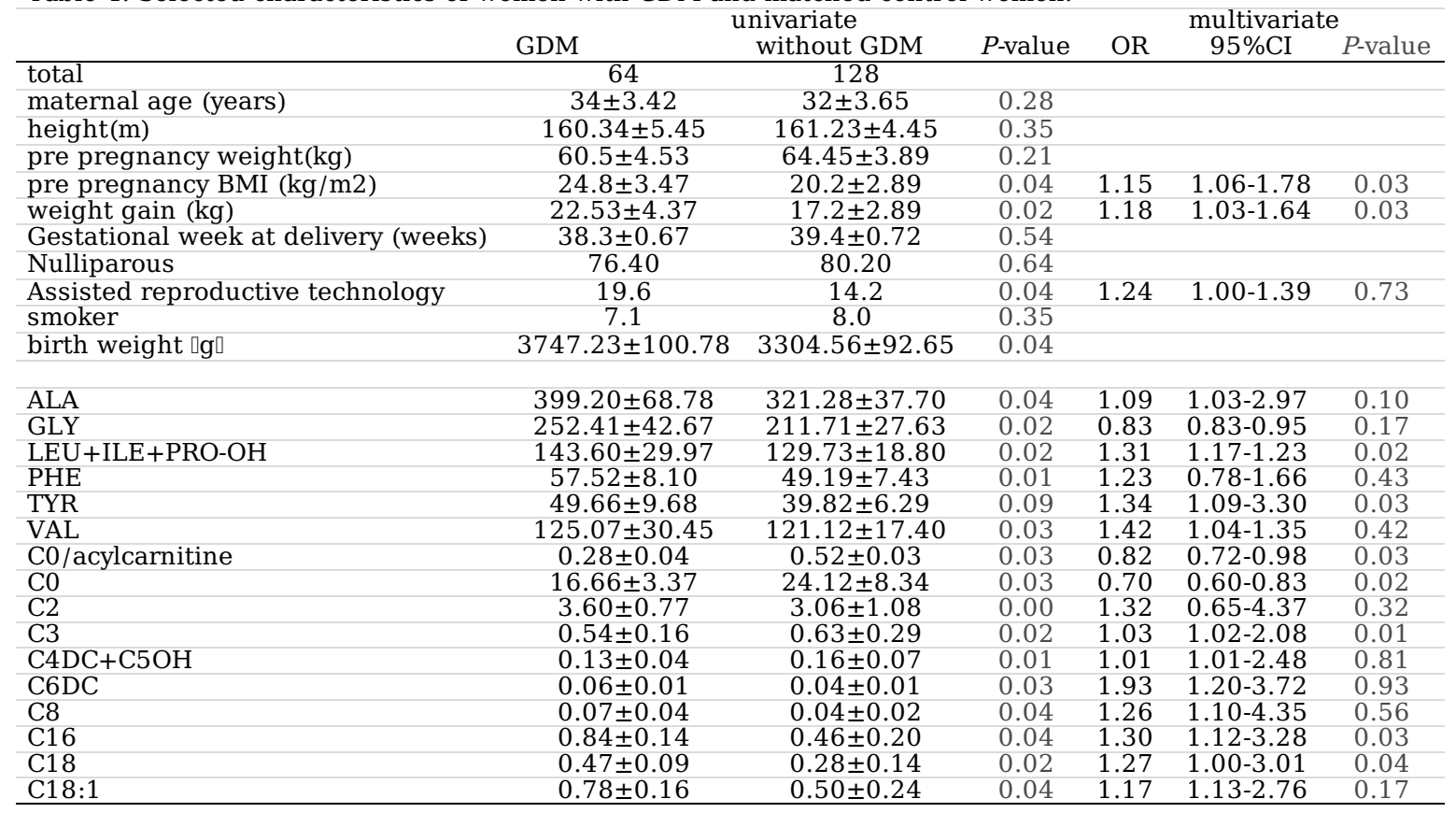

Table 5: Selected characteristics of GDM with macrosomia and non-macrosomia

\begin{tabular}{|c|c|c|c|c|c|c|}
\hline & macrosomia & $\begin{array}{l}\text { univariate } \\
\text { non-macrosomia }\end{array}$ & ${ }^{*} P$-value & OR & $\underset{95 \% \text { CI }}{\text { multivariate }}$ & ${ }^{*} P$-value \\
\hline total & 12 & 52 & & & & \\
\hline maternal age (years) & $34.98 \pm 4.68$ & $32.23 \pm 4.43$ & 0.79 & & & \\
\hline height(m) & $159.46 \pm 5.94$ & $161.46 \pm 5.95$ & 0.33 & & & \\
\hline pre pregnancy weight(kg) & $56.25 \pm 9.98$ & $52.42 \pm 7.84$ & 0.04 & 1.286 & $0.76-1.98$ & 0.64 \\
\hline pre pregnancy BMI $(\mathrm{kg} / \mathrm{m} 2)$ & $27.55 \pm 3.15$ & $24.21 \pm 3.02$ & 0.03 & 1.274 & $1.023-2.376$ & 0.03 \\
\hline weight gain $(\mathrm{kg})$ & $24.07 \pm 4.11$ & $18.42 \pm 4.10$ & 0.02 & 1.243 & $1.456-3.458$ & 0.04 \\
\hline Gestational week at delivery (weeks) & $38.4 \pm 0.78$ & $39.6 \pm 0.97$ & 0.83 & & & \\
\hline Nulliparous & 74.2 & 62.5 & 0.82 & & & \\
\hline Assisted reproductive technology & 19.6 & 14.2 & 0.55 & & & \\
\hline smoker & 7.4 & 8.2 & 0.96 & & & \\
\hline birth weight $\square g \square$ & $4247.52 \pm 100.78$ & $3297.34 \pm 98.65$ & 0.04 & & & \\
\hline ALA & $403.62 \pm 76.05$ & $393.62 \pm 66.82$ & 0.26 & & & \\
\hline GLY & $262.41 \pm 32.67$ & $241.71 \pm 30.75$ & 0.92 & & & \\
\hline $\mathrm{LEU}+\mathrm{ILE}+\mathrm{PRO}-\mathrm{OH}$ & $134.73 \pm 18.80$ & $119.60 \pm 19.97$ & 0.02 & 1.133 & $1.13-1.95$ & 0.81 \\
\hline PHE & $50.66 \pm 9.68$ & $48.82 \pm 6.29$ & 0.25 & & & \\
\hline TYR & $59.80 \pm 13.11$ & $47.19 \pm 7.42$ & 0.02 & 1.150 & $1.03-2.34$ & 0.03 \\
\hline VAL & $136.07 \pm 30.82$ & $121.12 \pm 19.40$ & 0.03 & 1.243 & 1.03 & 0.74 \\
\hline C0/actylcartine & $0.21 \pm 0.035$ & $0.32 \pm 0.035$ & 0.66 & & & \\
\hline $\mathrm{CO}$ & $10.68 \pm 3.28$ & $17.12 \pm 5.66$ & 0.03 & 0.759 & $0.50-0.87$ & 0.02 \\
\hline $\mathrm{C} 2$ & $3.64 \pm 0.76$ & $3.58 \pm 1.25$ & 0.03 & 1.157 & $1.02-3.37$ & 0.68 \\
\hline C3 & $0.43 \pm 0.17$ & $0.64 \pm 0.26$ & 0.02 & 1.167 & $1.12-1.98$ & 0.03 \\
\hline $\mathrm{C} 4 \mathrm{DC}+\mathrm{C} 5 \mathrm{OH}$ & $0.12 \pm 0.04$ & $0.14 \pm 0.05$ & 0.05 & & & \\
\hline C6DC & $0.06 \pm 0.01$ & $0.06 \pm 0.01$ & 0.77 & & & \\
\hline $\mathrm{C} 8$ & $0.08 \pm 0.04$ & $0.07 \pm 0.01$ & 0.54 & & & \\
\hline C16 & $0.86 \pm 0.12$ & $0.84 \pm 0.20$ & 0.03 & 1.154 & $1.03-2.42$ & 0.50 \\
\hline C18 & $0.50 \pm 0.09$ & $0.47 \pm 0.13$ & 0.05 & 1.167 & $1.24-3.23$ & 0.97 \\
\hline C18:1 & $0.52 \pm 0.16$ & $0.48 \pm 0.24$ & 0.55 & & & \\
\hline
\end{tabular}

\section{Figures}



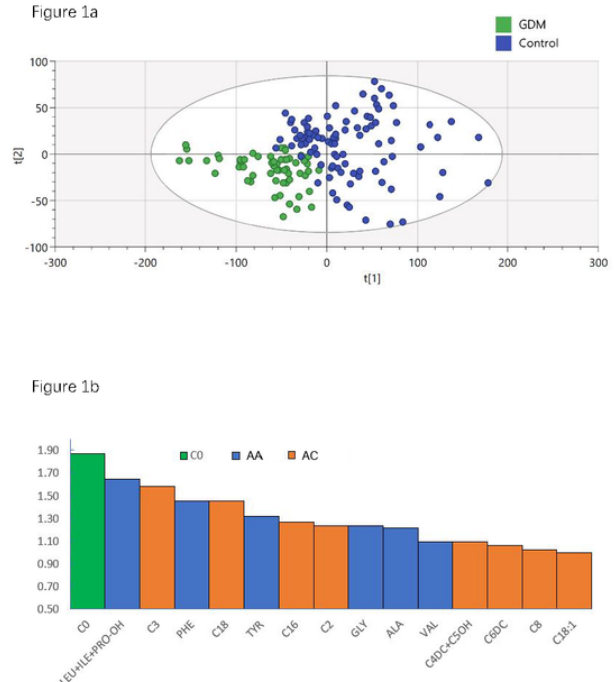

\section{Figure 1}

a) Partial least squares discrimination analysis (PLS-DA). Class separation is shown by loading plot, b) Variable of Importance for Prediction (VIP). Abbreviations: $A C$, acylcarnitine, $A A$, amino acids 


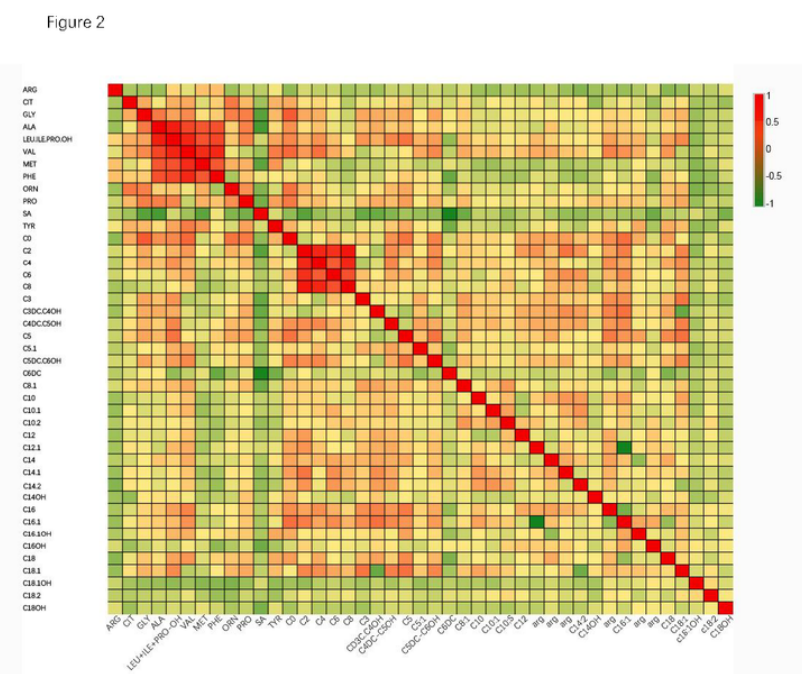

Figure 2

Correlation heatmap of all targeted metabolites. In red are two clusters of strongly intercorrelated metabolites. 


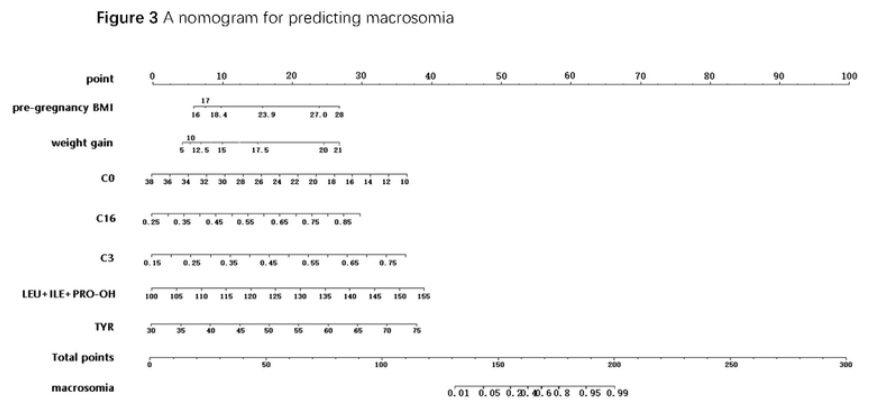

\section{Figure 3}

Carnitine-based nomogram for predicting macrosomia, predictor points (Points ${ }^{\wedge}$ scale; top) correspond to each variable. Points for all these variables are added and translated into the probability of macrosomia in GDM 